\section{Auch bei Asthmatherapie: Vertrauen ist gut - Kontrolle ist besser}

Heaney LG et al. Remotely Monitored Therapy and Nitric Oxide Suppression Identifies Non-Adherence in Severe Asthma. Am J Respir Crit Care Med 2018; doi:10.1164/rccm.201806-11820C

Bei Patienten mit schlecht zu kontrollierendem Asthma ist eine ungenügende Adhärenz eine mögliche Mitursache der fehlenden Krankheitskontrolle. Klinisch ist es schwierig, Patienten mit geringer Adhärenz und damit einem Potenzial für die Optimierung der Standardtherapie mit inhalativen Kortikosteroiden (ICS) von den Patienten zu unterscheiden, die wirklich ein refraktäres Asthma haben. Ein Telemonitoring könnte das ändern.

Liam G. Heaney vom Belfast City Hospital und seine britischen und irischen Kollegen untersuchten die Machbarkeit und den Effekt eines Suppressionstests des fraktionierten exhalierten Stickstoffmonoxids (FeNOsuppT) im klinischen Alltag in verschieden Zentren für schweres Asthma in Großbritannien. Die Patienten wurden gebeten, täglich das fraktionierte exhalierte Stickstoffmonoxid (FeNO) mit dem System NIOXvero zu messen und parallel für 7 Tage hochdosierte ICS $(1000 \mu \mathrm{g}$ Fluticason täglich) zusätzlich zu ihrer Standardmedikation mit ICS und langwirksamem Beta2-Agonisten (LABA) anzuwenden. NIOXvero ermöglicht webbasiert ein Fernmonitoring durch das betreuende Zentrum. Parallel erhielten die Patienten das System INCA (Inhaler Compliance Assessment), das an den DiscusInhaler angeschlossen werden kann, und akustisch dem Patienten übermittelt, ob richtig inhaliert wurde.

\section{Ergebnisse}

Von 250 Patienten beendeten 201 plangemäß die Studie. Bei 130 fand sich ein positiver FeNOsuppT (die FeNO-Werte wurden niedriger), bei 71 ein negativer FeNOsuppT. Zu Beginn der Intervention hatte sich das fraktionierte FeNO in den beiden Gruppen noch nicht unterschie- den. Bei positivem FeNOsuppT lag das FeNO am Ende im Median bei 26ppb (Interquartilrange [IQR] 16-36ppb), bei negativem bei 43ppb (IQR 38-73ppb). Im Vergleich zu den Patienten mit einem negativen Ergebnis identifizierte ein positiver FeNOsuppT also eine Population, die bei Adhärenz zur ICS/LABA-Therapie ein niedriges FeNO als Biomarker für eine gute Asthmakontrolle erreicht. Das spiegelte sich in der parallel verbesserten prozentualen Einsekundenkapazität wider: FEV1 \% lag im Mittel in den beiden Gruppen bei 88,2 ( $\pm 16,4)$ und 74,1 $( \pm 20,9)$ und war damit in der positiven Testgruppe signifikant höher als in der negativen $(p<0,01)$. Auch der Wert nach dem Asthma Control Questionnaire (ACQ-5) verbesserte sich in beiden Gruppen signifikant, bei positivem FeNOsuppT um im Mittel 1,2, bei negativem Testergebnis im Mittel um 0,9 Punkte.

FAZIT

Die fernüberwachte FeNO-Testung kann eine mangelnde Adhärenz als Grund für ein schlecht kontrolliertes Asthma aufdecken und bietet bei vielen Patienten die Möglichkeit, durch eine Optimierung der Standardtherapie die Asthmakontrolle zu verbessern. Das INCA-System kann zusätzlich gewährleisten, dass eine nicht beabsichtigte Nonadhärenz durch eine falsche Inhalationstechnik vermieden wird, betonten die Autoren.

Friederike Klein, München 